\title{
Density profile sensitivity study of ASDEX Upgrade ICRF Antennas with the TOPICA code
}

\author{
A. Krivska, ${ }^{\mathrm{a}, \mathrm{b}}$ S. Ceccuzzi, ${ }^{\mathrm{a}}$ D. Milanesio, ${ }^{\mathrm{c}}$ V. Bobkov, ${ }^{\mathrm{d}}$ F. Braun, ${ }^{\mathrm{d}}$ R. \\ Maggiora, ${ }^{\mathrm{c}}$ J.-M. Noterdaeme, ${ }^{\mathrm{d}, \mathrm{e}}$ and A. A. Tuccillo, ${ }^{\mathrm{a}}$ \\ ${ }^{a}$ Associazione EURATOM-ENEA sulla fusione, CR-Frascati, Frascati, Rome, Italy \\ ${ }^{b}$ Czech Technical University, Telecommunication Engineering Department, Prague, Czech Republic \\ ${ }^{c}$ Department of Electronics, Politecnico di Torino, Torino, Italy \\ ${ }^{d}$ Max Planck-Institut für Plasmaphysik, Euratom Association, Garching, Germany \\ ${ }^{e}$ University of Gent, EESA Department, Gent, Belgium
}

\begin{abstract}
During operation of the ASDEX Upgrade (AUG) ion cyclotron radio frequency (ICRF) system, Tungsten (W)-coated poloidal limiters and structures connected along magnetic field lines to the antenna can be sources of $\mathrm{W}$, which is attributed to sputtering by ions accelerated in radio frequency (RF) sheaths. In order to analyze and optimize the ICRF antenna performance, accurate and efficient simulation tools are necessary. TOPICA code was developed for analysis of ICRF antenna systems with plasma loading conditions modeled with 1D FELICE code. This paper presents an initial comparative analysis of two AUG ICRF antennas for a set of model plasma density profiles (with varying density gradient and antenna cut-off distance). The antennas are presently installed in AUG and differ in that one was partially optimized using HFSS code to reduce $E_{/ /}$near fields. Power transferred to plasma and sheath driving RF potentials are computed.
\end{abstract}

Keywords: ASDEX Upgrade; ICRF; TOPICA; Rectified potential; HFSS;

PACS: 52.25.Xz, 52.35.Hr, 52.50.Qt, 84.40.Ba, 52.40.Fd, 52.35.Hr, 52.35.Qz

http://www.aip.org/pacs/index.html

\section{INTRODUCTION}

During operation of the ASDEX Upgrade (AUG) ion cyclotron radio frequency (ICRF) system, Tungsten (W)-coated poloidal limiters and structures magnetically connected with antennas can be sources of W. The mechanism responsible for the $\mathrm{W}$ production is the sputtering by ions accelerated in radio frequency (RF) sheaths [1]. Within the simplest RF sheath models [2, 3, 4], ICRF antenna drives an oscillating RF potential $V_{R F}$ between the ends of open flux tubes in vicinity of the launcher. The RF potential is given by integration along the open magnetic field lines $V_{R F}=\int E_{/ /} d l$, where $E_{/ /}$is the RF parallel electric field. As a reaction to this RF potential, and due to the non-linear behaviour of the sheath at both flux extremities, the field line gets biased to a rectified DC potential. Ions are accelerated by this DC potential and hit plasma facing components at the end of the field lines $[5,6]$. This causes sputtering and leads to enhanced appearance of $\mathrm{W}$ during ICRF operation and possible contamination of the core plasma with $\mathrm{W}$. It can bring issues to fusion devices, which 
use or plan to use high- $Z$ materials for the first wall $[7,8]$ to withstand the high expected loads.

\section{TOPICA SIMULATIONS SET UP}

TOPICA (Torino Polytechnic Ion Cyclotron Antenna) code [9] was used to analyze the performances of the AUG ICRF antenna launchers. According to the code formulation, the fully $3 \mathrm{D}$ antenna, which is assumed to be in vacuum, is treated in spatial domain, while the spectral domain is adopted to evaluate the interaction with plasma.

AUG ICRF antennas are designed to operate over a wide range of frequencies (30$80 \mathrm{MHz}$ ). The fast magnetosonic wave is used for heating. Each of the four antennas consists of two toroidally spaced loops, which can be operated in-phase or out-ofphase. Two types of antennas, presently installed in AUG, are considered for our analysis (see Figure 1).

(a)

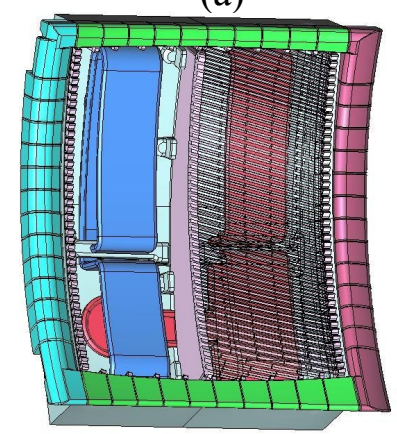

(b)

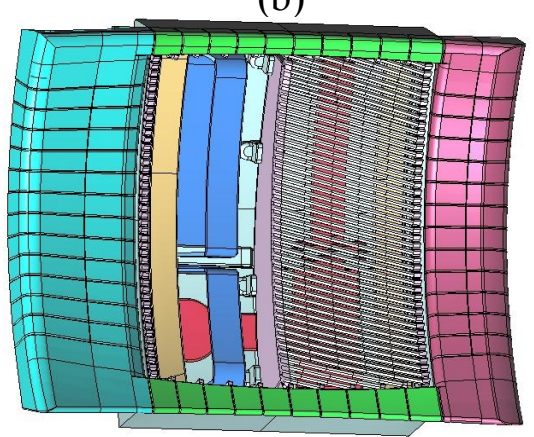

FIGURE 1. (a) Narrow ICRF antenna model and (b) wide antenna with thinner straps and asymmetric wider limiters.

These antennas differ in that the wide one has got wider limiters, bias-cut straps and additional poloidal plates. Wider limiters impose $E_{/ /}=0$ boundary condition close to the antenna and are meant to reduce $E_{/ /}$. The bias-cut straps with increased distance between them and protruding limiters are aimed to decrease image currents on the limiters. Additional poloidal plates are installed in order to carry and short-circuit fraction of poloidal currents [10].

As already alluded and documented by Figure 1, the fully detailed 3D geometries have been used for this comparison. In order to perform the simulations, antennas are placed into a recess and limited by plasma edge. Both the plasma edge, located $7 \mathrm{~mm}$ in front of the limiters, and the surface where the electric field (EF) map is calculated, $5 \mathrm{~mm}$ in front of the limiters, follow the curvature of the limiters; moreover, the same surfaces (in shape and in mesh density) are used for both antennas to minimize error sources. Few plasma scenarios with $3 \%$ of hydrogen minority in deuterium and static magnetic field $\mathrm{B}_{\mathrm{T}}=1.5 \mathrm{~T}, 11^{0}$ tilted with respect to the toroidal direction, were used. Simulations were performed for $\mathrm{f}=30 \mathrm{MHz}$ and $(0, \pi)$ phasing and for different density profiles with two cut-off antenna distances and two gradients (see Figure 2). 
(a)

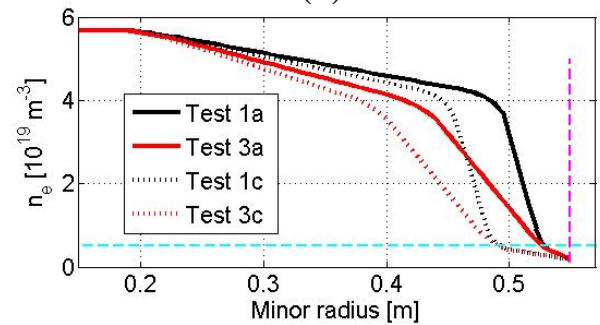

(b)

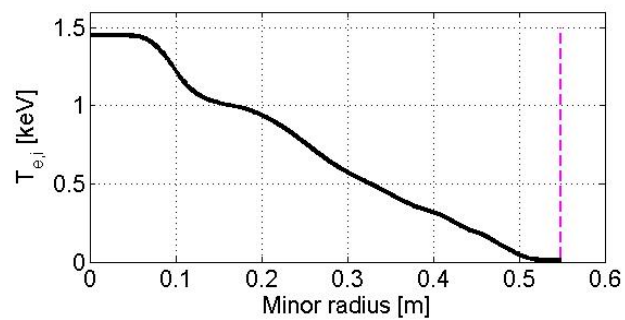

FIGURE 2. Density (a) and temperature (b) profiles used for simulations. Magenta lines represent position of the plasma edge.

\section{SIMULATIONS RESULTS}

Figure 3 reports the calculated coupled power for both antennas and all density profiles assuming $\mathrm{V}_{\max }=30 \mathrm{kV}$ in infinite coax. lines without any tuning and matching system. As expected, the transferred power is slightly higher for smoother gradient and smaller cut-off distance; the evanescent region, through which the fast magnetosonic has to travel, is shorter and more power is transferred to plasma. Coupled power is a bit lower for the wide antenna. Differences between antennas appear to be higher for smoother gradient and smaller antenna cut-off distance.

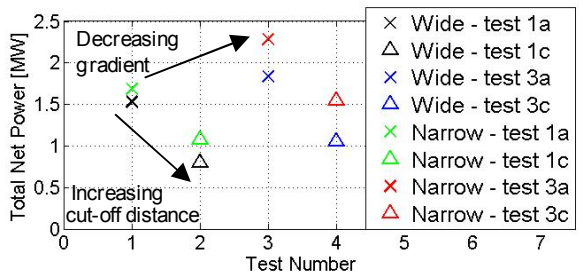

FIGURE 3. Calculated coupled power

Figures 4 and 5 show computed values of RF potential $a b s\left(V_{R F}=\int E_{/ /} d l\right)$ for all profiles and both antennas. RF potentials vary along vertical (poloidal) position; the peak values are situated in the lower and upper parts of both antennas, in correspondence of the top and bottom horizontal limiters, their shape being in good agreement with previous works $[1,11,12]$. For smaller antenna cut-off distance,RF potentials are slightly higher for bottom part of the narrow antenna. In other cases the curves seem to be rather similar. The comparison is only relative and does not give exact values. All computations were done with linear code and without considering any non-linear effects.

(a)

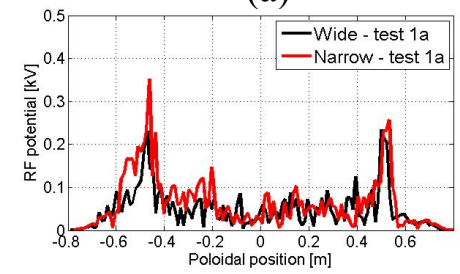

(b)

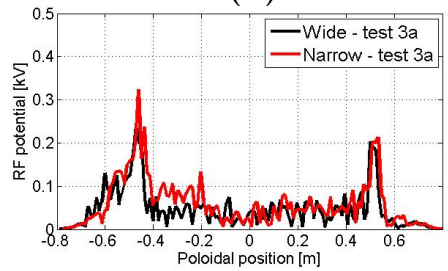

FIGURE 4. Gradient test - computed RF potential with constant antenna cut-off distance for steeper (a) and smoother (b) gradient, normalized to $1000 \mathrm{~kW}$ of coupled power. 
(a)

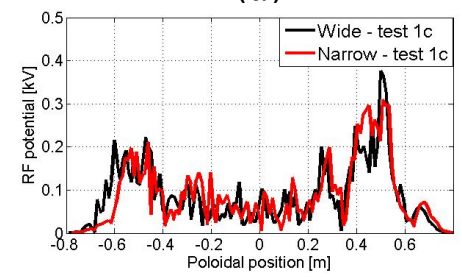

(b)

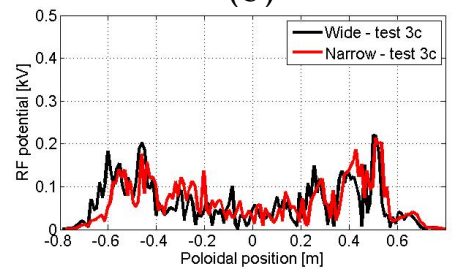

FIGURE 5. Cut-off distance test - computed RF potential with constant gradient for smaller (a) and bigger (b) antenna cut-off distance, normalized to $1000 \mathrm{~kW}$ of coupled power.

\section{CONCLUSION}

Computed values of the RF potentials and of the coupled power give indications of antenna performance and antenna's near field. Relative comparison of simulation results, for the given set of plasma parameters, show that the antenna with wide limiters couple slightly less power than the other one, while, in terms of RF potentials, the computed values of the two antennas look similar. A more detailed analysis on the antenna performance sensitivity, and on the role of the shape of the EF surface is ongoing, since differences appeared when comparing flat and curved geometries. More specifically, in case of flat approximated geometry, the simulation results show generally lower RF potentials for the wide antenna, which seems not to be the case when the curved geometry is adopted. To make a detailed comparison with the experiment, it would also be necessary to have appropriate diagnostics such as localized spectroscopy, infra red cameras and Langmuir probes.

\section{ACKNOWLEDGMENTS}

A. Krivska is supported by the ENEA international fellowship. S. Ceccuzzi and D. Milanesio are supported by the European Fusion Training Scheme „LITE“.

\section{REFERENCES}

1. V1. Bobkov et al., Nuclear Fusion 50, p. 035004 (2010)

2. V. Godyak and A. Kuzovnikov, Sov. J. Plasma Phys. 1, 276 (1975)

3. F.W. Perkins, Nuclear Fusion 29 p. 3 (1989)

4. J.R. Myra et al., Nuclear Fusion 30, p. 845 (1990)

5. L. Colas et al., EFDA Task TW6-TPHI-ICFS2 - Final Report "Faraday Shield RF modeling and RF sheath dissipation", 2007

6. L. Colas et al., Nuclear Fusion 45 p. 767-782 (2005)

7. R Dux et al., Journal of Nuclear Materials 363-365 (2007) 112

8. S. Wukitch et al., Journal of Nuclear Materials 363-365 (2007) 491

9. V. Lancellotti at al., Nuclear Fusion 46 476-499 (2006)

10. V. Bobkov et al., RADIO FREQUENCY POWER IN PLASMAS: Proceedings of the 18th Topical Conference. AIP Conference Proceedings, Volume 1187, pp. 125-132 (2009)

11. L. Colas et al., Journal of Nuclear Materials 363-365 (2007) 555-559

12. A. Mendes et al., Nucl. Fusion 50025021 (2010) 